\title{
Comparison between Actigraphic Records, the Evaluation of Behavior and Attention, and the Use of Psychostimulants in ADHD Patients: A Pilot Study
}

\author{
Clay Brites ${ }^{1}$, Talita Meneses de Almeida', Bruno S. B. Gonçalves², Gabriela M. Zuculo3, \\ Luciana Pinato ${ }^{3}$, Cristiane Bolfer ${ }^{4}$, Sylvia M. Ciasca ${ }^{1}$ \\ ${ }^{1}$ Department of Neurology, Laboratory of Learning Difficulties and Disabilities and Attention Disorders (DISAPRE-UNICAMP), \\ Campinas, Brazil \\ ${ }^{2}$ Department of School of Arts, Sciences and Humanity, São Paulo University (USP), São Paulo, Brazil \\ ${ }^{3}$ Department of Speech Therapy, Paulista State Universitiy (UNESP), Marília, Brazil \\ ${ }^{4}$ Department of Neurology, São Paulo University (USP), São Paulo, Brazil \\ Email: claybrites@gmail.com
}

How to cite this paper: Brites, C., de Almeida, T. M., Gonçalves, B. S. B., Zuculo, G. M., Pinato, L., Bolfer, C., \& Ciasca, S. M. (2017). Comparison between Actigraphic Records, the Evaluation of Behavior and Attention, and the Use of Psychostimulants in ADHD Patients: A Pilot Study. Psychology, 8, 1976-1987.

https://doi.org/10.4236/psych.2017.812126

Received: September 9, 2017

Accepted: October 16, 2017

Published: October 19, 2017

Copyright $\odot 2017$ by authors and Scientific Research Publishing Inc. This work is licensed under the Creative Commons Attribution International License (CC BY 4.0).

http://creativecommons.org/licenses/by/4.0/

\begin{abstract}
Introduction: Attention deficit hyperactivity disorder (ADHD) leads to cognitive problems in childhood and adolescence, and due to its associated hyperkinesias, it may also result in behavioral problems, which can lead to negative impressions amongst one's peers. Objective: To assess the impact of hyperkinesia reduction on the attention and behavior profiles of three children with the combined type of ADHD following an intervention featuring psychoactive medication. Methods: This pilot study was conducted at the Laboratory of Learning Difficulties and Disabilities and Attention Disorders (DISAPRE-UNICAMP). Objective data (including mean values of amplitude and frequency of movement over 24 hours) were obtained using an actigraph (a clock-like device), a behavioral assessment scale (the Child Behavior Checklist $[\mathrm{CBCL}]$ ), and the Strength and Weakness and ADHD Symptoms Normal Behavior (SWAN) rating scale (which was administered by clinicians and school staff members); these were cross-referenced to compare the children's performance before and after the use of psychoactive medication. The treatment was introduced after 15 days and maintained thereafter for 30 days, and the results were statistically analyzed by means of actigraphic variables (L5 and M10) and by student's $t$-test $(P<0.05)$. Results: There was a simultaneous reduction in the actigraphic index, the attention deficit scores of hyperactivity for both the CBCL and SWAN, and the somatic disorder symptom scores of the CBCL. These results were statistically significant when ex-
\end{abstract}


amining the pre- and post-medication data for each individual in the sample. Conclusion: The reduction in hyperkinesias in the three patients with ADHD resulted in improvements in inattention, somatic symptoms, and emotional symptoms.

\section{Keywords}

ADHD, SWAN Rating Scale, Actigraphy, CBCL

\section{Introduction}

Attention deficit hyperactivity disorder (ADHD) is a neurobiological development disorder that affects, on average, $3 \%-6 \%$ of children and adolescents. Its prevalence rate is about $5.29 \%$ and it is the most common neurobehavioral disorder in this age group and one of the most studied neuropsychiatric conditions in recent times (Castellanos \& Tannock, 2002; Barkley, 1997; Polanczyk, Lima, Horta, Biederman, \& Rohde, 2007). It is characterized by its chronic nature, and it features a predominantly polygenic etiology, which means that the affected individual shows signs and symptoms of attention deficits, high distractibility, hyperactivity, impulsivity, disorganization, and emotional inadequacy when faced with experiences that require self-regulation within the environment (Sobanski, Banaschewski, Sergeant, \& Faraone, 2010). The diagnosis is clinical in nature and based on the criteria set forth in the Diagnostic and Statistical Manual of Mental Disorders (DSM-IV); these criteria were recently updated in the DSM-5 (American Psychiatric Association, 1994; American Psychiatric Association, 2013).

These patients tend to experience adjustment difficulties when confronted with emotional conflicts; they do not tolerate rules, routines, repetition, or sudden changes in priorities, and they leave monotonous activities or responsibilities until the last minute. They avoid allocating mental effort to less pleasurable activities or to those without immediate reward, and these individuals often provoke discomfort among their peers by not assuming coherent positions exhibiting empathy impairments or by not adhering to routines of daily conduct. Additionally, they are often shy and withdrawn with new contacts or in new situations requiring flexibility and consideration of multiple strategies (Sobanski, Banaschewski, Sergeant, \& Faraone, 2010).

In this sense, the behavior of a person with the combined type of ADHD leads to chronic and recurrent difficulty in anticipating the demands of his or her surroundings; this triggers a process of maladjustment between their pace and the pace of the environment around them. Therefore, in this subtype, socio-affective detriments in the family and at school, externalized behavior, and sleep and eating disorders are present, while those with the inattentive subtype typically tend to manifest academic problems and internalized symptoms 
(Sobanski, Banaschewski, Sergeant, \& Faraone, 2010). These characteristics can be analyzed by structured behavioral assessment scales, which can be administered by those who live in closest contact with their children, parents and teachers. Some studies have shown that families who have children or adolescents with ADHD are busier, more stressed, and have a worse quality of life than those who do not have children with ADHD, as these children rely on their parents' frequent monitoring and caution to orient these children to follow rules and routines within the household environment and at school (Phelan, 2005; Marta, Rose, Laura, Jaume, \& Montse, 2011).

It is important to understand these patients' levels of attention deficit, unrest, and agitation, as well as their poor inhibitory control, as these generate destabilization. These symptoms are directly related to numerous stress factors and negative emotional symptoms, both at school and within the family. Adequate control of these behaviors has been highly related to quality of life improvements among the child and those around them, especially when pharmacological treatment with psychostimulants is introduced. In these instances, we see an almost immediate reduction in hyperactivity, excessive inattention, and other externalized symptoms (Brown et al., 2005; Haertling, Mueller, \& Bilke-Hentsch, 2015; Zelnik \& Terkel-Dawer, 2015). In order to objectively measure the hyperkinetic behavior of patients with combined ADHD, an actigraphic device, or an actimeter, can be used. It evaluates and analyzes, in real time, the amplitude and frequency of the child's excessive movements and swaying behaviors 24 hours a day, whether the child is in a structured environment or not. It uses actigraph variables to analyze a child's movement parameters, which are referred to as interdaily stability (IS), intradaily variability (IV), and the mean amplitude of M10 and L5. With respect to evaluations, behavioral scales are typically used; they are subjective in nature and employ natural observations. These scales are also related to the child's interactions with his or her peers. The scales have been validated and reliability supported by experience and evidence-based research on statistically secure sampling. For this study, the instruments used were the Child Behavior Checklist (CBCL) and the Strength and Weakness and ADHD Symptoms Normal Behavior (SWAN) rating scale, which are sensitive and suitable for checking global and attentional patterns of behavior, respectively; these scales may be applied by both parents and teachers.

The objective of this study is to verify the impact of hyperkinesias on the behaviors of the studied sample using two axes of evaluation: 1) the use of scales to evaluate the children's behaviors, as perceived by parents and teachers (these perceptions were then associated with and cross-checked against actigraphic registers); and 2) indices recorded by the actigraphic device, which was continuously worn by the children throughout the survey period. The impact of hyperkinesias on behavior can be assessed by peers' perceived reductions in the child's hyperactivity, as well as by monitoring the effects of this reduction on the child's productivity and attention. Moreover, these impacts can be determined if 
effective reductions are reflected by improvements in actigraphic indices and across sub-items on behavioral assessment scales.

\section{Materials and Methods}

Three patients diagnosed with the combined type of ADHD were recruited; these patients met the DSM- 5 criteria for this subtype of ADHD, and they were outpatients chosen from the Laboratory of Learning Difficulties and Disabilities and Attention Disorders (DISAPRE-UNICAMP). Males and females between the ages of $8-13$ years, those who did not receive any type of previous treatment or intervention, and those in middle or lower socioeconomic classes were selected. All of these participants were invited to sign the informed consent form (ICF-CNS 466/12), and only then were they included in the study.

Inclusion criteria: 1) Children with a confirmed diagnosis of the combined type of ADHD, as established by the DSM-5 criteria; 2) parental consent and signature of the ICF by the same; and 3) children between 7 and 12 years of age.

Exclusion criteria: 1) Children and adolescents with epilepsy, learning disorders, or any other neurological condition that provokes movement restrictions due to postural overload or motor sequel, such as cerebrovascular accidents and scoliosis; 2) children being treated with anti-histamines or neuroleptic or psychotropic medication; 3) children aged 13 years older or those below 7 years of age; and 4) children with an allergic or idiosyncratic reaction to any medication that will be used in this study.

Following submission to, and upon receiving approval from, the Research Ethics Committee, protocol number 159/2010, the children began their participation in the study. The children received a formal diagnosis of ADHD, either the hyperactive or combined type, by a clinical team, as based on established diagnostic protocols. Then, the informed consent forms (per the resolution of the National Health Council-CNS 466/12), were signed by the responsible parties and sent from the database at DISAPRE/UNICAMP. The subjects included in the study are described below:

Subject 1 is a male patient, 8 years and 10 months of age, who was a single-born, full-term infant, and who was adequate weight for gestational age at birth without perinatal complications. The patient comes from a low-middle socioeconomic status. He attends public school in the municipality of Santa Bárbara d'Oeste (SP), where he is in his fourth year of elementary school, and has achieved good academic standing. He was diagnosed with the combined type of ADHD.

Subject 2 is a female patient, 11 years and 1 month of age. She is the first-born of two children, and she was carried to term without perinatal intercurrences. She is from a middle-class family, and she is attends private school in Campinas (SP). She is currently in her seventh year of elementary school II and has achieved good academic standing. She was diagnosed with the combined type of ADHD. 
Subject 3 is a 12-year-old male patient. He is an only child, and he comes from a lower socioeconomic status. He is a public school student in Mairinque (SP), where he is currently in his seventh year of elementary school II. He was diagnosed with the combined type of ADHD.

Three children and adolescents, aged 8, 11, and 13 years old, and each with a diagnosis of the combined subtype of $\mathrm{ADHD}$, were evaluated during the study. They were instructed to use an actigraphic device for a period of 45 days. This group of children was not treated during the first 15 days of the study; however, in the 30 days thereafter, these children were subsequently treated with psychoactive medication.

The children were evaluated using two instruments: the CBCL, which was completed by the children's parents or responsible caregivers (this version of the scale was suitable for children aged 6 - 18 years), and the SWAN rating scale, which specifically measures attentional abilities. Both scales were administered at time zero by the author of this study, and they were subsequently administered by a certified psychologist on the $40^{\text {th }}$ day of the study. The SWAN rating scale was also completed by the children's school counselors and teachers. The teachers and counselors administered these instruments at time zero (during the non-medicated period of the study) and 45 days later (30 days after the medication was first administered) in the school environment.

The CBCL was administered to the children and their parents by experienced professionals, and the data were analyzed using specific CBCL software. Children and adolescents were classified based on the test results, and the findings were analyzed by the children's age, using the general American population's results on this scale as a control. The SWAN rating scale, in turn, was administered by experienced medical professionals (SWAN-C) and by teachers or school coordinators (SWAN-E) to the patients' parents, and the data were analyzed by the author in accordance with the scale parameters.

For the actigraphic records, the Act Trust (clock-like) device developed by Condor Instruments was used ActStudio software. The participants were instructed on how to use the device, and they were provided with the guidance necessary to avoid imbalances and/or deviations in the results. The device was placed and fixed on the upper, non-dominant limb, like a watch. Said instructions followed the 2002 Protocol of Practical Parameters for the use of an actigraph, according to Littner et al. (2003). All participants would wear the device 24 hours a day, for 45 days. The device contains mechanisms that prevent the individual from disabling or modifying its operation. The device is activated via its specialized software program, and the recording time of the device's registration was determined by the research team.

The records for each participant were subsequently analyzed, computed, and recorded in specific software that featured a registry of containing the specific measures recorded by the actigraph, as well as counters and circadian variables. The circadian variables include IS, IV, and the mean amplitude of M10 and L5. 
IV allows us to report on rhythm fragmentations in daily activity. Its calculation is based on the first derivative of the grouping of the actigraph data on an hourly basis, and it is represented by the following equation:

$$
I V=\frac{\sum_{i=2}^{N}\left(X_{i}-X_{i-1}\right)}{(N-1) \sum_{i=1}^{N}\left(X_{i}-X_{m}\right)^{2}}
$$

where the first derivative is the result of the subtraction between previous movement elements $\left(X_{i-1}\right)$ and subsequent elements $\left(X_{i}\right)$ from the untreated data. From this first derivative, the root mean square is calculated by $\sum_{i=2}^{N}\left(X_{i}-X_{i-1}\right)^{2} /(N-1)$, and the results are normalized by the population variance, $\sum_{i=1}^{N}\left(X_{i}-X_{m}\right)^{2}$. Ample time periods are required to analyze how the amount of daily sleep and wake times increase the IV value.

IS provides information on the synchronization between rest-activity rhythms and the light-dark cycle, and it is calculated based on the average values of a 24-hour day. IS can be represented by the following equation:

$$
I S=\frac{\sum_{h=1}^{P}\left(X_{h}-X_{m}^{2} N\right)}{(P) \cdot \sum_{i=1}^{N}\left(X_{i}-X_{m}\right)^{2}}
$$

where $N$ is the total number of data obtained, $\mathrm{p}$ is the number of data per day (24 points in this case), $X_{m}$ is the average of all obtained data, $X_{h}$ provides a profile of the average hour, and $X_{i}$ represents each hour in sequence. The IS, in short, is calculated as the variance of the average daily profile, divided by the total variance.

Nocturnal activity, measured by the nonparametric variable L5, reflects the average activity that occurs in the 5 hours featuring the lowest amount of movement for the day. Diurnal activity, in turn, is measured by variable M10; it is a variable that reflects the average activity of the 10 most active hours in a given day. High M10 values are found in people with a very restless or hectic lifestyle.

The calculation of the difference between nocturnal and diurnal activity generates values that highlight the amplitude of the activity-rest rhythm. Low-amplitude values are frequently observed in individuals with certain medical conditions, such as Alzheimer's disease. Furthermore, those with high amplitudes are assumed to be in better health. The amplitude of the rest-activity cycle (RA) can be verified by means of the following formula:

$$
R A=\frac{M 10-L 5}{M 10+L 5}
$$

Retrospectively, the data were analyzed using SPSS; furthermore, the L5 and M10 variables were analyzed offline. The differences in the measured parameters between the non-medicated and medicated phases were statistically analyzed using analysis of variance (ANOVA). In addition, the differences between times were compared and subsequently correlated to the circadian variables; they were also cross-checked by means of multiple regression, using clinical data such as 
gender and age, whether the children were treated or not, and whether the children were in a state of sleep or wakefulness, etc. The technical variations in the calibration of the apparatus were respected.

In order to reduce the signs of hyperkinesia, a psychoactive medication (long-acting methylphenidate; Ritalin) was chosen. A dose of $10 \mathrm{mg}$ of the medication was administered twice daily, at 8 a.m. and $1 \mathrm{p} . \mathrm{m}$. The children were not given the treatment at night. This daily dose was adequate for all individuals in this study, as their weights and heights were similar. The medication was introduced after 15 days of non-treatment, and the children continued to receive it for 30 days. The data produced by the scales and by the actigraph were cross-checked before and after medication use. In order to determine whether there were any differences between the means of the total scale score and the $\mathrm{T}$ Score indices before and after the medication was taken, Student's paired $t$-test was applied, and the significance level was set to $P=0.05$.

\section{Results}

A reduction in the actigraphic indices was confirmed overall (Table 1), and there was also a corresponding reduction in the attention deficit and hyperactivity scores across both the CBCL and SWAN scales. Furthermore, there was a decrease in the CBCL symptoms associated with somatic disorders (Table 2). These results were statistically significant for each individual included in this study, after pairing the sample data both pre- and post-medication administration. Table 1 shows the mean, median, and standard deviations of the indices for the Total Score and T score scales, and the respective $P$-values for Student's paired $t$-test.

In the three patients studied, the actigraphic records revealed a reduction in daytime and nighttime hyperkinesia, even during sleep, when comparing the records obtained between the pre- and post-medication stages. As shown in Table 1, the M10 data decreased, on average, by $91.99 \%$ among the three patients in the study after introducing the psychoactive medication. Additionally, an average reduction of $65.89 \%$ was observed across the L5 parameters. Of note, only the variations in L5 were statistically significantly relevant, indicating that there was a greater reduction in hyperkinesias during rest (i.e., during sleep) (Table 1).

Table 1. Indexes of the actigraphacs registers (L5 e M10 pre and post medication).

\begin{tabular}{|c|c|c|c|c|c|c|}
\hline & \multirow{2}{*}{$\mathrm{L}^{\star}$ pre } & \multirow{2}{*}{ L5 ${ }^{\star}$ post } & \multirow{2}{*}{$\begin{array}{l}\text { L5 Variation } \\
\text { (before/after) }\end{array}$} & \multirow{2}{*}{ M10 ${ }^{* *}$ pre } & \multirow{2}{*}{$\mathrm{M} 10^{* *}$ post } & \multirow{2}{*}{$\frac{\text { M10 Variation }}{\text { (before/after) }}$} \\
\hline & & & & & & \\
\hline P1 & 949.46 & 723.77 & $76.23 \%$ & 196,326 & $183,408.2$ & $93.42 \%$ \\
\hline P2 & 714.77 & 475.69 & $66.55 \%$ & $165,703.9$ & $141,832.2$ & $85.59 \%$ \\
\hline P3 & 548.15 & 300.92 & $54.90 \%$ & $212,848.2$ & $206,417.3$ & $96.97 \%$ \\
\hline General average & 737.46 & 5001.267 & $65.89 \%$ & 191,626 & $177,219.2$ & $91.99 \%$ \\
\hline
\end{tabular}


Table 2. Averages, medium and standard deviations (Total Score e T Score) of the CBCL and SWAN indexes and the actigraphics variations pre and post medication.

\begin{tabular}{|c|c|c|c|c|c|c|c|c|}
\hline \multirow[b]{2}{*}{ CBCL indexes } & \multirow[b]{2}{*}{ Scales } & \multicolumn{3}{|c|}{ Pre medication } & \multicolumn{3}{|c|}{ Post medication } & \multirow[b]{2}{*}{$p$-value } \\
\hline & & Average & Medium & $\begin{array}{l}\text { Standard } \\
\text { deviation }\end{array}$ & Average & Medium & $\begin{array}{l}\text { Standard } \\
\text { deviation }\end{array}$ & \\
\hline Anxious/ & Total score & 7.00 & 8.00 & 1.73 & 5.67 & 6.00 & 2.52 & 0.54 \\
\hline Depression & $\mathrm{T}$ score & 63.00 & 66.00 & 5.20 & 59.67 & 60.00 & 6.51 & 0.57 \\
\hline Insight/ & Total score & 5.33 & 6.00 & 1.15 & $4.00 \backslash$ & 2.00 & 3.46 & 0.53 \\
\hline Depression & T score & 66.67 & 66.00 & 3.05 & 61.33 & 58.00 & 7.57 & 0.38 \\
\hline Somatic & Total score & 1.67 & 2.00 & 1.53 & 2.33 & 0.00 & 4.04 & 0.85 \\
\hline Complaints & $\mathrm{T}$ score & 56.00 & 57.00 & 5.58 & 57.33 & 50.00 & 12.70 & 0.90 \\
\hline Social & Total score & 8.33 & 8.00 & 5.51 & 6.00 & 6.00 & 3.00 & 0.25 \\
\hline Problems & $\mathrm{T}$ score & 67.67 & 67.00 & 14.01 & 62.00 & 62.00 & 8.00 & 0.24 \\
\hline Thinking & Total score & 3.00 & 3.00 & 1.00 & 3.00 & 2.00 & 2.65 & 1.00 \\
\hline Problems & T score & 58.33 & 58.00 & 4.51 & 57.33 & 54.00 & 8.50 & 0.78 \\
\hline Attentional & Total score & 16.33 & 15.00 & 2.31 & 12.00 & 12.00 & 3.00 & $0.04^{\star *}$ \\
\hline Problems & T score & 85.67 & 83.00 & 8.33 & 71.00 & 73.00 & 6.24 & $0.03^{\star *}$ \\
\hline Oppositor & Total Score & 5.67 & 7.00 & 3.21 & 3.00 & 1.00 & 4.36 & 0.43 \\
\hline Behavior & T score & 63.00 & 63.00 & 8.00 & 55.33 & 52.00 & 7.57 & 0.37 \\
\hline Agressive & Total score & 14.33 & 13.00 & 8.08 & 12.67 & 10.00 & 11.23 & 0.73 \\
\hline Behavior & T score & 67.33 & 66.00 & 11.06 & 65.00 & 61.00 & 16.37 & 0.75 \\
\hline Affective & Total score & 6.67 & 6.00 & 4.04 & 4.67 & 3.00 & 3.78 & 0.22 \\
\hline Problems & T score & 70.33 & 68.00 & 4.04 & 62.67 & 60.00 & 8.33 & 0.10 \\
\hline Anxious & Total score & 2.33 & 2.00 & 0.58 & 2.33 & 2.00 & 1.53 & 1.00 \\
\hline Problems & T score & 57.33 & 58.00 & 2.08 & 57.33 & 58.00 & 6.03 & 1.00 \\
\hline Somatics & Total score & 3.00 & 2.00 & 1.73 & 0.00 & 0.00 & 0.00 & 0.09 \\
\hline Problems & $\mathrm{T}$ score & 64.00 & 61.00 & 5.20 & 50.00 & 50.00 & 0.00 & $0.04^{* *}$ \\
\hline ADHD & Total score & 11.00 & 11.00 & 1.00 & 9.67 & 9.00 & 4.04 & 0.58 \\
\hline Symptoms & T score & 71.67 & 72.00 & 1.53 & 68.67 & 68.00 & 11.01 & 0.67 \\
\hline Oppositional-Defiant & Total score & 5.33 & 6.00 & 3.05 & 4.33 & 2.00 & 4.93 & 0.68 \\
\hline Symptoms & T score & 63.00 & 66.00 & 9.85 & 61.00 & 52.00 & 16.46 & 0.80 \\
\hline Conducts & Total score & 7.33 & 8.00 & 6.03 & 4.67 & 3.00 & 3.79 & 0.38 \\
\hline Problems & T score & 63.67 & 69.00 & 10.11 & 60.00 & 60.00 & 6.00 & 0.63 \\
\hline \multirow{2}{*}{ SWAN } & $C^{* * *}$ & 26.00 & 26.00 & 4.00 & 19.33 & 21.00 & 3.79 & $0.04^{* *}$ \\
\hline & $E^{* * * *}$ & 24.33 & 27.00 & 5.51 & 11.00 & 9.00 & 9.16 & 0.07 \\
\hline $\mathrm{L}^{1}$ & & 737.46 & 714.77 & 201.61 & 500.13 & 475.69 & 212.48 & $<0.01^{\star *}$ \\
\hline $\mathrm{M} 10^{2}$ & & $191,626.00$ & $196,326.00$ & $23,920.98$ & $177,219.20$ & $183,408.20$ & $32,734.33$ & 0.11 \\
\hline
\end{tabular}

${ }^{\star} p$-value Wilcoxon test to paired data; ${ }^{* *} p$-value $<0,05$, there are significant diferences between the before and after means; ${ }^{* *}$ SWAN-C: scale applied for

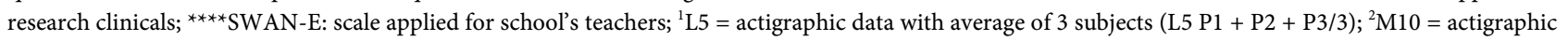
data with average of 3 subjects (M10 P1 + P2 + P3/3). 
With respect to the results obtained on the CBCL (Table 2), after the introduction of the psychoactive medication, there was a demonstrated reduction in the behavioral inadequacy indices for both internalizing and externalizing symptoms, but statistical significance was only found for somatic problems and attention problems. Upon studying the results of the SWAN scale (Table 2), a significant reduction of ADHD symptoms was observed, but only when analyzing the results of the scale administered by clinical staff (SWAN-C).

\section{Discussion}

The overall evaluation of the results showed that the use of psychoactive medication among the three patients studied herein resulted in reduced hyperkinesia. The existing literature is extensive and redundant when demonstrating the effectiveness and quick action of methylphenidate in patients with ADHD, as this medication reduces associated symptoms and actigraphic indices, while improving quality of life scores or scores that reflect activities in the classroom or labschool environment (De Crescenzo et al., 2014; Uebel et al., 2016; Haynes, Lopez-Romero, \& Anand, 2015). In fact, some research emphasizes how there are decreases in inattentive indices, increases in performance during structured activities, and improvements across neuropsychological tests when medication administration is measured with the use of an actigraph unit, as in the case of monitoring medication use while implementing the Continuous Performance Test (CPT) (Edebol, Helldin, \& Norlander, 2013).

Both in parallel and comparatively, the behavioral assessment scales and the specific scale for ADHD (SWAN) also showed a significant reduction in the scores related to somatic problems and attention deficits in all individuals in the study sample, especially when assessed by clinicians in a double-blind scenario; when the scale was administered by the school, such statistical value was not observed. The use of scales by teachers and non-specialists naturally raises concerns due to the lack of experience and the lack of expertise required to translate observations into clinical parameters. Moreover, the difficulty faced while assessing the intensity of a particular behavior may explain the lack of comparative significance when the SWAN scale is applied by teachers and school staff members (SWAN-E). However, in this study, precautions were taken to account for the competence of those administering the survey, as well as to ensure that these individuals were being well prepared to take part in the study; they were additionally guided by the clinical team that conducted the study. This scale is easy to use and it is accessible for application by education professionals; it can also be administered for various study purposes within the realm of ADHD (Brites, Salgado-Azoni, Ferreira, Lima, \& Ciasca, 2015), although it is important to note that when the SWAN scale was applied in a double-blind scenario by the clinical team (SWAN-C), there was a significant reduction in the attention deficit score.

When the data were matched at an individual level, and comparisons were made between the phase in which the children received medication and the 
phase in which they did not, we can conclude that for each individual in the study, the reduction in hyperkinesias during times of rest had improved the children's inattention and somatic problems. The association between excessive restlessness and attention loss is directly proportionate, which is in accordance with the current findings in the literature. Furthermore, the actigraphic records are similar for patients with either the inattentive type of ADHD or those with the combined type (Dane, Schachar, \& Tannock, 2000). Obviously, we cannot draw the same conclusion when considering the three patients together, as the sample is obviously inadequate. Similarly, we cannot definitively affirm that the psychoactive medication was solely responsible for this effect. Even so, it appears that the pharmacological effect was really the only constant variable that was altered during the survey period, and the introduction of the same seemed to have solely influenced the results reported here. In addition, the validity and reliability of the actigraphic methodology for recognizing ADHD, which was achieved by analyzing the behavioral profile (observations) and objective records (rapid oscillations) of the three children in a naturalistic environment, was able to give the obtained data a higher level of veracity when paired with the behavioral results expressed in the scales (Kam et al., 2010; Gonçalves, Adamowicz, Louzada, Moreno, \& Araújo, 2015). This was the case even in relatively short periods of time (15 - 40 days), which was in accordance with current chronobiological models.

In this pilot study, the use of psychoactive medication, and the consequent reduction of hyperkinesias, was not sufficient enough to reduce the negative impact of ADHD on behavior in general; however, these methods were proven to be effective for improving somatic symptoms and attention-related problems. It is important to note that during this study, the patients remained under observation for 45 days; if this model were extended for a greater period of time, the outcomes may have differed, despite the evidence that indicated how short intervals of measurement are often sufficient for observing clinical results (Martin-Martinez et al., 2012). Conversely, this fact may also have contributed to the finding that the negative perceptions held by family members and school staff members toward the hyperkinesias observed in this sample were not effectively or convincingly modified. Of note, individuals exhibiting these types of behavioral disorders often do not see modified perceptions and evaluations from their peers for a long period of time after any behavioral improvements first appear; it may simply be the case that these observations may suffer from subjective interference.

\section{Conclusion}

As such, this study showed a direct relationship between the reductions in actigraphic indices and improvements in somatic symptoms and attention deficits following the introduction of psychoactive medication. However, due to the small sample size employed in this investigation, the results were not sufficient enough to infer and conclude that medication use, as a single variable, was effec- 
tive in reducing either the children's inappropriate behaviors or their actigraphic records. We are aware of methodological restrictions.

However, this paper could serve as model to new researches with big samples and future statistical excellence. Besides, it can be used to clinical analyses more objective for medication evaluation on ADHD assessment.

\section{Acknowledgements}

I would like to thank the parents of these patients, who provided their consent and allowed their children to participate in this study. I would also like to express my gratitude to those schools that allowed their professors to contribute to this endeavor. Special thanks to the Emeritus Prof. Dr. Joseph A. Sergeant, from the Vrije Universitait of Amsterdam, who provided his guidance when developing the initial basis for this study, and who inspired the author to embark on this important approach to research on ADHD. English-language editing of this manuscript was provided by Journal Prep.

\section{References}

American Psychiatric Association (1994). Diagnostic and Statistical Manual of Mental Disorders DSM-IV (4th ed.) Washington: American Psychiatric Association.

American Psychiatric Association (2013). Diagnostic and Statistical Manual of Mental Disorders DSM-5 (5th ed.) Washington: American Psychiatric Association. https://doi.org/10.1176/appi.books.9780890425596

Barkley, R. A. (1997). Behavioral Inhibition, Sustained Attention, and Executive Functions: Constructing a Unifying Theory of ADHD. Psychological Bulletin, 121, 65-94. https://doi.org/10.1037/0033-2909.121.1.65

Brites, C., Salgado-Azoni, C. A., Ferreira, T. L., Lima, R. F., \& Ciasca, S. M. (2015). Development and Applications of the SWAN Rating Scale for Assessment of ADHD: A Literature Review. Brazilian Journal of Medical and Biological Research, 48, 965-972. https://doi.org/10.1590/1414-431X20154528

Brown, R. T., Amler, R. W., Freeman, W. S., Perrin, J. M., Stein, M. T., Feldman, H. M. et al. (2005). Committee on Quality Improvement, Subcommittee on ADHD. Pediatrics, 115, e749-e757. https://doi.org/10.1542/peds.2004-2560

Castellanos, F. X., \& Tannock, R. (2002). Neuroscience of Attention-Deficit/ Hyperactivity Disorder: The Search of Endophenotypes. Nature Reviews Neuroscience, 3, 617-628. https://doi.org/10.1038/nrn896

Dane, A. V., Schachar, R. J., \& Tannock, R. (2000). Does Actigraphy Differentiate ADHD Subtypes in the Clinical Research Setting? JAACAP, 39, 752-760. https://doi.org/10.1097/00004583-200006000-00014

De Crescenzo, F., Armando, M., Mazzone, L., Ciliberto, M., Sciannamea, M., Figueroa, C. et al. (2014). The Use of Actigraphy in the Monitoring of Methylphenidate versus Placebo in ADHD: A Meta-Analysis. ADHD Attention Deficit and Hyperactivity Disorders, 6, 49-58. https://doi.org/10.1007/s12402-013-0122-x

Edebol, H., Helldin, L., \& Norlander T. (2013). Measuring Adult Attention Deficit Hyperactivity Disorder Using the Quantified Behavior Test Plus. PsyCh Journal, 2, 48-62. https://doi.org/10.1002/pchj.17

Gonçalves, B. S. B., Adamowicz, T., Louzada, F. M., Moreno, C. R., \& Araújo, J. F. (2015). 
A Fresh Look at the Use of Nonparametric Analysis in Actimetry. Sleep Medicine Reviews, 20, 84-91. https://doi.org/10.1016/j.smrv.2014.06.002

Haertling, F., Mueller, B., \& Bilke-Hentsch, O. (2015). Effectiveness and Safety of a Long-Acting, Once-Daily, Two-Phase Release Formulation of Methylphenidate (Ritalin LA) in School Children under Daily Practice Conditions. DHD Attention Deficit and Hyperactivity Disorders, 7, 157-164. https://doi.org/10.1007/s12402-014-0154-X

Haynes, V., Lopez-Romero, P., \& Anand, E. (2015). Attention-Deficit/Hyperactivity Disorder under Treatment Outcomes Research (AUTOR): A European Observational Study in Pediatric Subjects. ADHD Attention Deficit and Hyperactivity Disorders, 7, 295-311. https://doi.org/10.1007/s12402-015-0177-y

Kam, H. J., Shin, Y. M., Cho, S. H., Kim, S. Y., Kim, K. W., \& Park, R. W. (2010). Development of a Decision Support Model for Screening Attention-Deficit Hyperactivity Disorder with Actigraph-Based Measurements of Classroom Activity. Applied Clinical Informatics, 1, 377-393. https://doi.org/10.4338/ACI-2010-05-RA-0033

Littner, M., Kushida, C. A., Anderson, W. M., Bailey, D., Berry, R. B., Davila, D. G. et al. (2003). Practice Parameters for the Role of Actigraphy in the Study of Sleep and Circadian Rhythms: An Update for 2002. Sleep, 26, 337-341.

https://doi.org/10.1093/sleep/26.3.337

Marta, G., Rose, N., Laura, M., Jaume, M., \& Montse, V. (2011). The Disorder for Deficit of Attention with Hyperactivity: The Effect in the Context Family. ADHD-Attention Deficit and Hyperactivity Disorders, 3, 198.

Martin-Martinez, D., Casaseca-de-la-Higuera, P., Alberola López, S., Andres Llano, J., López-Vilallobos, J. A., Ardura-Fernandez, J., \& López-Alberola, C. (2012). Nonlinear Analysis of Actigraphic Signals for the Assessment of the Attention-Deficit/Hyperactivity Disorder (ADHD). Medical Engineering \& Physics, 34, 1317-1329. https://doi.org/10.1016/j.medengphy.2011.12.023

Phelan, T. W. (2005). ADD/ADHD: Attention Deficit Hyperactivity Disorder. São Paulo: M. Books.

Polanczyk, G. V., Lima, M. S., Horta, B. L., Biederman, J., \& Rohde, L. A. (2007). The Worldwide Prevalence of ADHD: A Systematic Review and Metaregression Analysis. The American Journal of Psychiatry, 164, 942-948. https://doi.org/10.1176/ajp.2007.164.6.942

Sobanski, E., Banaschewski, T., Sergeant, J. A., \& Faraone, S. (2010). Emotional Liability in Children and Adolescents with ADHD: Clinical Correlates and Familial Prevalences. Journal of Child Psychology and Psychiatry, 51, 915-923. https://doi.org/10.1111/j.1469-7610.2010.02217.x

Uebel, H., Albrecht, B., Kirov, R., Haise, A., Döpfner, M., Freisleder, J. et al. (2016). What Can Actigraphy Add to the Concept of Labschool Design in Clinical Trials? Current Pharmaceutical Design, 16, 2434-2442. https://doi.org/10.2174/138161210791959845

Zelnik, N., \& Terkel-Dawer, R. (2015). The Clinical Profile of Children with ADHD That Require OROS-Methylphenidate Combined with Shorter-Acting Formulations. ADHD Attention Deficit and Hyperactivity Disorders, 7, 313-318.

https://doi.org/10.1007/s12402-015-0168-Z 